\title{
LPG in perfluorinated GI-POF for concentration measurement in liquids
}

\author{
J. Castrellon-Uribe ${ }^{1,2}$, M. Lomer ${ }^{1 *}$, H.S.G. Roufael ${ }^{1}$, L. Rodriguez-Cobo ${ }^{1}$ and J.M. Lopez-Higuera ${ }^{1}$ \\ ${ }^{1}$ Photonics Engineering Group, Cantabria University, Av. De los Castros s.n. 39005 Santander, Spain \\ ${ }^{2}$ Centro de Investigación en Ingeniería y Ciencias Aplicadas, CIICAp, Universidad Autónoma del \\ Estado de Morelos, UAEM, Av. Universidad 1001, Col. Chamilpa, Cuernavaca Morelos, México. \\ *lomerm@unican.es
}

\begin{abstract}
This paper describes the manufacture of long period gratings (LPG) for liquid concentration sensing. The LPGs are made in perfluorinated polymer optical fiber point to point using a pulsed laser at $355 \mathrm{~nm}$. A LPG of period $75 \mu \mathrm{m}$ and a wavelength of resonance of $910 \mathrm{~nm}$ is used for measurement of water with different concentrations of sugar.

OCIS codes: (060.2370) Fiber optics sensors
\end{abstract}

\section{Introduction}

Long period gratings in optical fibers have a broad application domain. In optical telecommunications include bandpass filters, tunable filters, variable attenuators, mode converters and dispersion compensators [1-3]. In sensor technology are used in the measurement of pressure, temperature, strain, measurement in structures or refractive index of liquid [4-6]. Most work on LPGs has been performed in silica optical fibers due to the availability in optical communications. LPGs in polymer optical fibers (POF) have small attention due to having higher propagation loss silica fibers as well as the difficulty of connecting extremities components not yet standardized. However, in recent years there have been important advances in new structures and new materials for POF, particularly fibers with cores perfluorinated (PF), which is an excellent material and have low loss in the region of the visible and infrared spectrum. Good that these fibers have been manufactured to work in high-speed communications [7]. They can be interesting in applications in sensor technology, particularly because of its low value of core refractive index (about $1.35)$.

To date, very few studies of LPGs in POF have been reported. A LPG written in material PF flattened obtained from removing the cladding from the fiber cylindrical structure was reported by Liu et al. [8, 9]. The authors reported a diffraction efficiency of $1.6 \%$ and a modulation index of photoinduced $3 \times 10^{-4}$ and concluded inability to written grating directly in the core. On the other hand, the LPGs in POF commercially available are lacking in most studies fiber Bragg grating (FBG) in the POF have been made based cores poly-methyl-methacrylate (PMMA) doped with other monomers or colorants prepared by the same authors [10,11].

This paper first presents the fabrication of LPGs in PF-GI-POF using UV pulsed laser to wavelength of $355 \mathrm{~nm}$. The fiber used in this work is commercially available and has a ratio of diameter core/cladding of 120/500 $\mu \mathrm{m}$. The LPGs are performed on the lateral side of the cladding fiber and written point by point. This fabrication method allows the modulation index variation of the cladding controlling the grooves depth. The fabricated LPGs are used to measure sugar concentration in water and check the influence of the external environment. Experimental results are presented and discussed.

\section{Fabrication LPG in perflurinated POF}

The experimental setup of fabrication is shown in Figure 1 (a) consists of a UV pulsed laser wavelength $355 \mathrm{~nm}$ obtained from the third harmonic of Nd: YAG laser. The pulses have a frequency of $10 \mathrm{~Hz}$. The laser beam is focused by a lens on a lateral side of the cladding fiber. The fiber is located in a V-shaped groove on a PMMA block and integral with a sliding plate micrometer. Writing the grating on the lateral side of the fiber is performed point by point and longitudinal displacement along the axis. The system is optimized in power and exposure time on the polymer fiber to the LPG writing either by ablation of the outer region of the cladding. LPGs are characterized online by a white light source and optical spectrum analyzer (OSA). In the preliminary work have used two types PF-GI-POF, GI-POF50 and GI-POF1200 and manufactured by Chromis Fiberoptics ${ }^{\circledR}$ with a diameter ratio core/cladding of 50/500 and $120 / 500 \mu \mathrm{m}$, respectively. The refractive indices of the core and cladding are about 1.35-1.34. The LPGs made in the fiber core into account the index difference between core/cladding to determine the resonance wavelength [12]. In this work, the LPGs affect only cladding the fiber, therefore the approximation to determine the length of resonance may take into account the equation given by Zhu et al. [13]. 
Figure 1(b) shows the picture of a LPG fabricated in a PF-GI-POF with core diameter of $120 \mu \mathrm{m}$ by the method described in this paper. The period of the grating is $75 \mu \mathrm{m}$ and a length of $7.5 \mathrm{~mm}$. Each groove of the grating have been written with an energy of $75 \mathrm{~mJ}$ and for $30 \mathrm{~s}$. As seen in the picture, the grooves are quite regular and fiber aspect is preserved very well. Figure 1 (c) shows the transmission spectrum of the fiber before and after the manufacture of the LPG. We can see a resonance wavelength well defined at $910 \mathrm{~nm}$ and amplitude rejection of 14 $\mathrm{dB}$. Most important values of the amplitude of rejection can be obtained by controlling the number periods of the LPG.

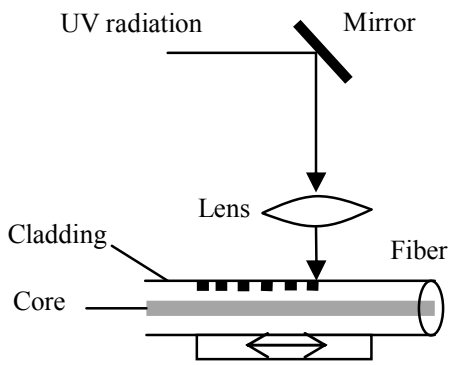

(a)

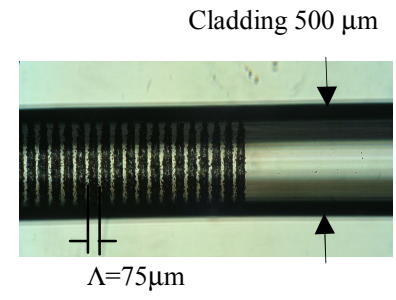

(b)

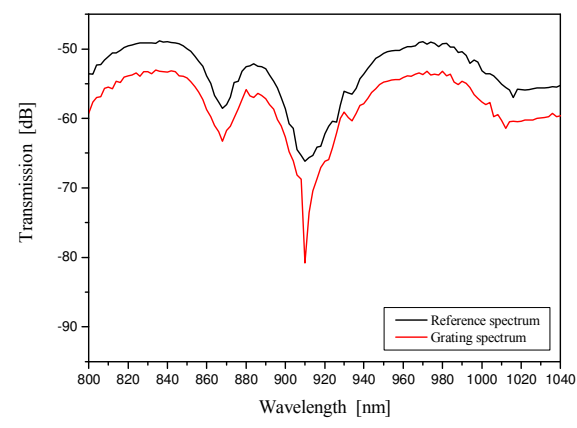

(c)

Figure 1. LPGs en PF-GI-POF, (a) experimental setup for fabrication, (b) Photo of perfluorinated fiber LPG 500 cladding diameter, and (c) Transmission spectrum of the fiber before and after fabrication.

\section{Experimental results of the concentration measurement}

As is well known, LPG in general is sensitive to the surrounding medium, temperature, strain, curvature or refractive index. With the aim of studying the response of the LPG to small refractive index variation given by variations in fluid concentration for example, the LPG has been immersed in water with different concentrations of sugar. In Figure 2 is plotted the results of the measurements. One can see that the resonance wavelength reference (a) is shifted to shorter wavelengths when immersed in water (b) then to larger wavelengths where water has a sugar concentration of $5 \mathrm{~g} / 1$ (c) and $10 \mathrm{~g} / 1(\mathrm{~d})$. All measurements were performed at constant temperature $\left(21^{\circ} \mathrm{C}\right)$. These results are consistent with the theory [13], since water has an index lower refractive index (1.33) below the cladding (1.34). When water contains sugar refractive index of fluid increases, and if the sugar concentration increases, the refractive index can exceed the rate of the cladding. Resonance peaks $3.5 \mathrm{~nm}$ moving a sugar concentration of $5 \mathrm{~g} / 1$ (c) and $6.5 \mathrm{~nm}$ for a concentration of $10 \mathrm{~g} / 1(\mathrm{~d})$.

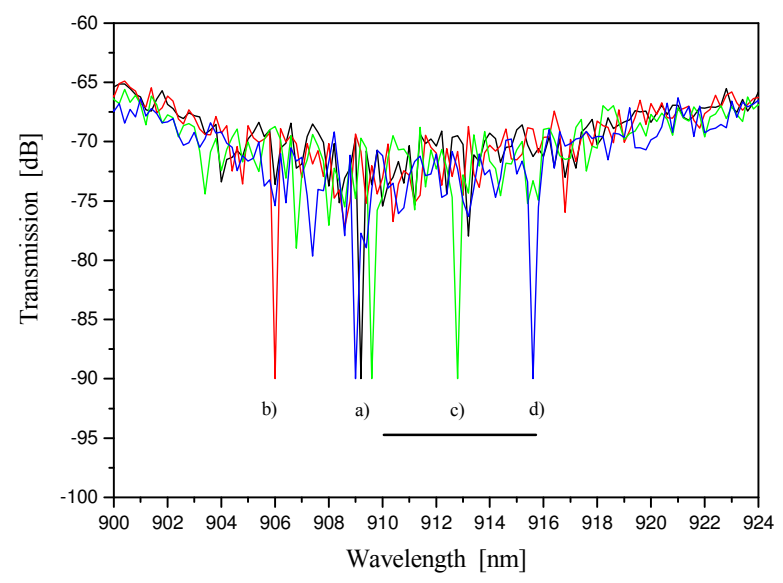

Figure 2. Spectral response of LPG with period $\Lambda=7.5 \mu \mathrm{m}$ and length $75 \mu \mathrm{m}$ when immersed in water with different concentrations of sugar. a) $\lambda \sim 909 \mathrm{~nm}$; reference grating, b) $\lambda=906 \mathrm{~nm}$; water, c) $\lambda=912.8 \mathrm{~nm} ; 5 \mathrm{~g} / 1$ sugar, d) $\lambda=915.6 \mathrm{~nm} ; 10 \mathrm{~g} / 1$ sugar. 


\section{Conclusion}

Have demonstrated the fabrication of LPG in PF-GI-POF using a UV pulsed laser $(\lambda=355 \mathrm{~nm})$. The grating is written point by point on a lateral side of the cladding of the fiber without affecting the fiber core. Our fabrication method can be an alternative for LPGs with PF-GI-POF commercially available. The concentration's measure of sugar in water has been used to show high sensitivity to the external environment of the LPGs. An additional advantage of the LPGs in PF-GI-POF is the low refractive index of the fiber and can be supported for the sensing of the other liquids.

\section{Acknowledgement}

This work was supported by the MCYT (Spain), project CICYT: TEC2010-20224-C02-02 and CONACyT

(Mexico) by sabbatical stay at the University of Cantabria, file: 186243.

\section{References}

[1] Hill, K.O., Malo, B., Vineberg, K., Bilodo, F., Johnson, D., and Skinner, I., "Efficient mode conversion in telecommunication fiber using externally written gratings,' Electron. Lett. 26, 1270-1272 (1990).

[2] Lee, K.S., Erdogan, T., "Fiber mode conversion with tilted gratings in an optical fiber”, J. Opt. Soc. Am. A 181176 (2001).

[3] D.B. Stegall, D.B., Erdogan, T., "Dispersion control with use of long-period fiber gratings”, J. Opt. Soc. Am. A 17, 304 (2000).

[4] Liu, Y., Zhang, L., Bennion, I., "Fibre optic load sensors with high transverse strain sensitivity based on long-period gratings in B/Ge co doped fibre", Electron. Lett. 35, 661 (1999).

[5] James, S.W., Tatam, R.P., “Optical fibre long-period grating sensors: characteristics and application”, Meas. Sci. Technol. 14 R49 (2003).

[6] Ding, J.F., Zhang, A.P., Shao, L.Y, Yan, J.H., and He, S., "Fiber-Taper Seeded Long-Period Grating Pair as a Highly Sensitive RefractiveIndex Sensor", IEEE Photon. Technol. Lett. 17, 1247-1249, (2005).

[7] Ishigure, T., Nihei, E., and Koike, Y., “Graded-index polymer optical fiber for high-speed data communication”, Appl. Opt. 33, 4261-4266 (1994).

[8] Liu, H.Y., Peng, G.D., Chu, P.L., Koike, Y. and Watanabe, Y., "Photosensitivity in low-loss perfluoropolymer (CYT'OP) fibre material", Electron. Lett. 37, 347-348 (2001).

[9] Liu,H.Y., Peng, G.D., Chu, L.P., “Thermal stability of gratings in PMMA and CYTOP polymer fibers”, Opt. Commun. 204, 151-156 (2002).

[10] Li, Z., Tam, H., Xu, L., and Zhang, Q., "Fabrication of long-period gratings in poly (methyl methacrylate-co-methyl vinyl ketone-co-benzyl methacrylate)-core polymer optical fiber by use of a mercury lamp," Optics letters 30, 1117-1119 (2005).

[11] Luo, Y., Li, Z., Zheng, R., Chen, R., Yan, Q. Zhang, Peng, G.D., Ming, H., and Zhu, B., "Birefringent azopolymer long period fiber gratings induced by $532 \mathrm{~nm}$ polarized laser," Optics Communications 282, 2348-2353 (2009).

[12] Vengsarkar,A.M., Lemaire, P.J., Judkins, B.J., Bhatia, V., Erdogan, T., and Sipe, J.E., "Long period fiber gratings as band rejection filters," J. Lightwave Technol., 14, 58-64, (1996).

[13] Zhu, T., Rao, Y-J., Wang, J-L., and Song, Y., "A highly sensitive fiber-optic refractive index sensor based on an edge-written long-period fiber grating", IEEE Photon. Technol. Lett., 19, 1946-1948 (2207) 